\title{
Repercusiones de las macro-políticas de industrialización por sustitución de importaciones y de innovación en la dinámica urbana de los Barrios Bajos, Valdivia'
}

\section{Aftermath of the macro-policies of Industrialization for Import Substitution and Innovation in the urban dynamics of the Barrios Bajos, Valdivia}

\author{
Laura Rodríguez Negrete² (1), Tirza Barría Catalán³ (1) \\ y Robinson Silva Hidalgo 4
}

\begin{abstract}
RESUMEN
La discusión respecto de la política y el espacio es un debate pendiente y, al mismo tiempo, urgente en las ciudades no metropolitanas chilenas. En función de dar respuesta a esta necesidad el artículo explora las repercusiones de las macro-políticas de sustitución de importaciones y de innovación en la espacialidad de los Barrios Bajos, Valdivia y su efecto transformador en el corto, pero también en el largo plazo. El marco temporal utilizado corresponde desde el inicio del siglo XX hasta 1973 y luego desde el inicio del nuevo milenio a la fecha. La metodología incorpora diversas fuentes tales como; una revisión bibliográfica exhaustiva, bases de datos de financiamientos del Estado para proyectos en el área de la innovación, salidas a terreno, las cuales procesa a través de un cuadro de síntesis y una cartografía. Además, se realiza un registro fotográfico y entrevistas en profundidad, para ilustrar en detalle algunos aspectos de la investigación. Se establece como resultados que, siendo las macro políticas -acuerdos convenidos por la elite- estas traspasan los gobiernos de turno y en esto radica el efecto dinamizador para la ciudad contemporánea. Las macro-políticas transforman los Barrios Bajos de Valdivia en un proceso lento, pero sostenido en el tiempo; y se adecuan a las necesidades materiales requeridas por dichas políticas, bajo una conceptualización que denominamos de adaptación política-espacial convivencial.
\end{abstract}

Palabras clave: Políticas, barrios en transformación, dinámica urbana, Valdivia.

Investigación desarrollada en el marco del Proyecto ANID "Anillo de Ciencias Sociales, SOC1403, Patrimonio industrial: formas de habitar colectivo en el sur de Chile. Aportes para su puesta en valor y recuperación integrada" 


\begin{abstract}
Politics and space is a pending debate, as well as, it is urgent to discuss it at the non-metropolitan Chilean cities. In order to answer this requirement, this article explores the macro-politics of industrialization by substitution of imports and innovation's aftermath in the spatiality of Barrios Bajos's neighborhood, Valdivia and it transforming effect in the short, but also in the long term. The period used corresponds from the beginning of the 20th century to 1973 and then from the beginning of the new millennium up to date. The methodology incorporates different sources such as an exhaustive bibliographic review, innovation projects funded by the State database, field trips. All this was sort out through making a summary table and a cartography. In addition, to illustrate some detailing aspects of the investigation, it carries out a photographic record and in-depth interviews. As a result, we establish that, being the macro policies - agreements made by the elite - they pass the ongoing governments and this is why they are gravitating for the contemporary city. Macro-policies transform the neighborhood through a slow, but sustained process. It adapts to the policies' required material needs, under a conceptualization that we call "political-spatial occupation coexistence".
\end{abstract}

Keywords: Policy, changing neighborhoods, urban dynamic, Valdivia.

La estrategia para el posicionamiento de las políticas de industrialización por sustitución de importaciones (ISI) y de innovación en el desarrollo urbano de Chile revive la atención sobre el espacio y la política. Este debate permite resaltar la proximidad existente entre la política y las transformaciones urbanas, especialmente cuando se trata de estrategias macro-políticas llevadas a cabo por el impulso del Estado, pero con la anuencia del sector privado, e insertas en una red regional transnacional (en este caso Latinoamérica). Así al menos lo confirma Silva (2007) en el caso de la política de industrialización por sustitución de importaciones y Moya Muñoz y Molina Jara (2017) en el caso de la innovación. En función de lo anterior esta investigación explora las repercusiones de las macro-políticas de industrialización por sustitución de importaciones (ISI) y de innovación en la espacialidad de los Barrios Bajos, Valdivia y su efecto transformador en el corto, pero también en el largo plazo. El marco temporal utilizado corresponde desde el inicio del siglo XX hasta 1973 y luego desde el comienzo del nuevo milenio a la fecha. Se formula la siguiente hipótesis, las repercusiones de las macro-políticas de industrialización por sustitución de importaciones y de innovación se manifiestan en pequeñas adaptaciones del espacio urbano, las cuales conviven con elementos urbanos dependientes de anteriores modelos ideológicos a modo de un palimpsesto. Siendo los Barrios Bajos de Valdivia, un caso de estudio que evidencia lo anterior.

Las repercusiones de estas macro-políticas toman tiempo para manifestarse y abordar los problemas de los sistemas que están reemplazando, consiguiendo materializarse como modelos más adelante. Traspasan la administración de gobiernos en específico y se instalan dentro de una perspectiva general de desarrollo de una región (en este caso Latinoamérica) tal como se verá más adelante. Se trata de una visión paradigmática, sin duda acuñada por la elite política, y que en ningún caso obedece a las inquietudes manifestadas por la ciudadanía, aun cuando las visiones en algunos casos contrapuestas, pueden correr de forma paralela. Ejemplos de lo anterior corresponden a la macro-política de industrialización por sustitución de importaciones (Ortega Martínez, 2012; Hurtado Ruiz-Tagle, 1966). Proyecto Autoritario Neoliberal con (Hidalgo \& Janoschka, 2014; Mattos, 1999; Taylor, 2006). Tal como la reciente estrategia de la innovación con (Cruz, 2008; Moya Muñoz \& Molina Jara, 2008).

Mientras las macro-políticas de industrialización por sustitución de importaciones (comienzos del siglo XX hasta 1973) y el proyecto autoritario neoliberal (1973-1990), han desencadenado 
variados estudios de su influencia en el espacio urbano $y$, en particular, de las transformaciones derivadas de estos procesos, especialmente en Santiago de Chile durante el siglo XX y lo que va del siglo XXI; hasta ahora ha existido una escasa preocupación por las transformaciones en el largo plazo, en barrios de ciudades no metropolitanas. De la misma manera, existe una nula preocupación por los impactos de la nueva macro-política de la innovación en el sistema urbano del país, tal como se observa más adelante, donde las pocas referencias a esto último solo consideran a la ciudad de Santiago. Para cerrar esta brecha, la literatura en espacio urbano y política puede ganar significativamente en perspectivas producidas desde la investigación, en los efectos transformadores de los barrios, en el corto, tal como en el largo plazo.

Por otro lado, la racionalidad de explorar estas dos macro-políticas está dado, en primer lugar, porque siendo el aparato estatal, con sus múltiples recursos financieros, logísticos, humanos y técnicos, un factor de peso en la fuerza de penetración de estas macro-políticas, el Estado no actúa solo. Se produce una alineación de las fuerzas conductoras de los distintos poderes, en este caso el legislativo y sobretodo el empresarial (Casanova Brito, 2019). Para el caso de la macro-política de innovación existe un similar escenario (Moya Muñoz \& Molina Jara, 2017). Por este motivo la macro-política emanada de la dictadura chilena (1973-1990) quedará fuera del alcance de esta investigación. Aun cuando el ejecutivo en aquella época operó con pleno acuerdo del empresariado para llevar a cabo las siete modernizaciones del Estado, el poder legislativo estuvo ausente por el estado de excepción, en el cual se gobernó durante los 17 años (Hidalgo et al., 2016). No obstante, es insoslayable la referencia constante al poder transformador de la dictadura sobre la sociedad chilena y el espacio, torciendo el curso del destino urbano y agregando múltiples características al territorio, impactos presentes y patentes hasta la actualidad (Pérez, 2017). En segundo lugar, el sector investigado, los Barrios Bajos, corresponde a un sector peri-central de la ciudad. Este se estructura bajo dos tipologías que definen un sector de barrio obrero y uno de los industriales, tal como se verá más adelante en la descripción del sector. Esta condición lo situó de manera relevante en la época de industrialización por sustitución de importaciones, tal como lo argumenta Silva Hidalgo (2020), y ahora adquiere lentamente una relevancia en la macro-política de innovación.

Al examinar el efecto transformador de las macro-políticas en los barrios, este artículo se enfocará en particular en la estructura de la vivienda y la concepción del espacio público -integrando las circulaciones- junto con las instalaciones esenciales para el desempeño de estas macro-políticas (industrias, servicios, instalaciones educativas u otras). Por lo tanto, este artículo se concentrará en específico en la dimensión espacial y su dinámica en los ámbitos ya mencionados, en vez de proporcionar una visión general de sus amplias repercusiones. Considerando que Sánchez destaca la complejidad y diversidad de temas relativos a la dinámica urbana en un número especial de la Revista Geografía Norte Grande.

Los centros urbanos son recipientes de poderosas dinámicas. Dichas fuerzas son ejercidas por numerosos actores que en una constante interacción, tensión y conflicto van estableciendo y modificando la morfología y estructura de los asentamientos. Lo anterior evidencia la complejidad del espacio urbano, donde esta investigación incorpora una perspectiva del fenómeno (2013: 5).

El artículo se organiza en tres etapas; en la primera parte, se presentan las investigaciones relativas a caracterizar histórica y espacialmente las macro-políticas de industrialización por sus- 
titución de importaciones y de innovación. En la segunda parte, se identifican espacialmente las transformaciones urbanas a partir de las conceptualizaciones constitutivas de las macro-políticas en los Barrios Bajos, Valdivia y, en la tercera parte, se analizan las repercusiones de corto y largo plazo de estas macro-políticas.

\section{Metodología}

La investigación se llevó a cabo inicialmente a través de una revisión bibliográfica exhaustiva, con el propósito de caracterizar las políticas de industrialización por sustitución de importaciones (ISI) y la de innovación. Si bien es cierto que las repercusiones de ambas macro-políticas atañen a un amplio espectro del quehacer nacional, el alcance de la revisión está circunscrito al ámbito urbano-espacial. Además, se revisó el Diario El Correo de Valdivia entre los años 1930 y 1960, para complementar la revisión bibliográfica. Se consideró estas fechas, ya que la vivienda obrera comienza a ser un elemento urbano de importancia relativa en Valdivia, a partir de la década de 1930 (Almonacid Zapata, 2000).

En segundo lugar, se revisaron distintas bases de datos referidas al financiamiento destinado a innovación; entre estas están la CORFO (2010-2017), SERCOTEC con el programa Crece Industria Creativa, Capital de Emprendimiento Cultural, Capital Semilla y CONICYT, a través de FONDEF. Dicha información fue recabada a través de la plataforma de Transparencia. Con lo anterior se realizó una matriz con la siguiente información: programa gubernamental, nombre beneficiario y ubicación geográfica. Esta información, sirve exclusivamente para elaborar la cartografía, de manera de ubicar los lugares en el mapa de los Barrios Bajos.

En una tercera instancia se realizaron sucesivas salidas a terreno para identificar las direcciones de los beneficiarios en el territorio, junto con realizar un registro fotográfico de las instalaciones mencionadas como parte de la política de innovación. Aquí se utiliza la fotografía, tal como es empleada por Iturra Muñoz en el recorrido urbano

La continua evolución del proceso de observar, es rápidamente incorporado en la discusión del fenómeno urbano permitiendo pensar y hacer al mismo tiempo, problematizando mientras se observa y generando conocimiento mientras se representa (2015: 24)

La salida a terreno también permitió localizar geográficamente algunos servicios, tales como los relativos al ocio, hospedaje, gastronomía y otros vinculados a la macro-política de innovación, los cuales forman parte del proyecto de desarrollo local no declarado por parte de las agencias estatales orientadas al financiamiento de este tipo de proyectos. A partir de todo anterior se elabora el cuadro $\mathrm{N}^{\circ} 2$, sintetizando las principales características urbanas a considerar. Es decir, este cuadro se elaboró considerando la revisión bibliográfica, el diario El Correo, las salidas a terreno y la data de los proyectos financiados por todas las entidades del Estado.

En una última etapa se seleccionó a dos beneficiarias del proyecto CORFO, ubicado en el barrio, para realizar una entrevista en profundidad. Esto último solo con el propósito de ilustrar el proceso y dar a conocer aspectos de carácter cualitativos que beneficiaran una comprensión más profunda del fenómeno. 


\section{Discusión y resultados}

\section{Las macro-políticas y su dimensión espacial}

La perspectiva de esta investigación da cuenta del hecho de que las macro-políticas están situadas no solo en el tiempo, sino también en el espacio y repercuten en ambos. Su alcance geográfico no se circunscribe a los límites nacionales, sino que traspasa las fronteras y se articula dentro de un orden transnacional. Entonces decimos que el impulso creador, sostenedor y también eliminador - por qué no decirlo- de las macro-políticas radica no solo al interior del país, sino que es parte de un contexto internacional que las conduce.

Chile, al igual que los otros países latinoamericanos independizados de la Corona española, y luego de casi prácticamente un siglo de vida republicana, necesitaba incorporarse a la modernidad y un claro rasgo de esto fue la creación de diversas macro-políticas, una de ellas la de industrialización por sustitución de importaciones (ISI). Los distintos autores concuerdan en aspectos como la crisis del modelo exportador de recursos mineros y agrícolas y la crisis del modelo parlamentario como respuesta al surgimiento de esta macro-política de industrialización por sustitución de importaciones (Hurtado Ruiz-Tagle, 1966; Ortega Martínez, 2012; Silva, 2007).

En consideración a las necesidades del nuevo Estado desarrollista, era necesario incorporar la industrialización interna como motor de desarrollo del sector público, pero también del sector privado. La fuerte articulación entre los discursos del progreso, la educación y la industria no se hicieron esperar y vieron su ejecución en la creación de la CORFO en 1939.

En lo inmediato, su creación fue obra del gobierno de Pedro Aguirre Cerda, y tuvo como objetivo fundamental elaborar políticas económicas y crediticias y un plan de fomento de la producción que hicieran posible el desarrollo de la economía en general y de la industria en particular (CORFO, 2013: 14).

Este impulso dado por el Estado chileno al fortalecimiento de la industria tuvo como primera repercusión la fuerte migración del campo a la ciudad. Esto trajo como primera consecuencia la necesidad de vivienda para los amplios contingentes de personas que iban a residir a la ciudad en la búsqueda de mejores condiciones de vida y de un porvenir más próspero. Así, las industrias, junto con una serie de iniciativas del Estado, comenzaron el lento proceso de encontrar una solución habitacional a los que lo requerían. Consecuentemente surgieron otras necesidades: educación, salud, higiene. Respecto de este proceso, la literatura lo sitúa dentro de un paradigma bastante especifico, el cual, de acuerdo con Bárbara de Vos Eyzaguirre, establece que:

Desde la gran crisis de 1930 hasta fines de la década de 1960, la idea eje que domino la política económica chilena y que pasó a constituir una verdadera tarea nacional, abordada por todos los sectores de la sociedad, fue lograr el desarrollo del país mediante un proceso de industrialización (1999: 11).

En este texto, la autora detalla la dimensión de los recursos, organizaciones y exenciones tributarias a los que los industriales tuvieron acceso para insertarse dentro de este paradigma. Por otra parte, Carmen Cariola Sutter y Osvaldo Sunkel (1982), hacen un gran énfasis en el incremento 
urbano-poblacional que trajo el crecimiento sostenido de las principales ciudades del país. Este experimentó grandes transformaciones, que fueron especialmente relevantes en las ciudades más grandes; además de Santiago; tal fue el caso de Concepción (Aliste, et al., 2012).

Otros autores centraron su atención en la dinámica espacial de la ciudad bajo este modelo, (Borsdorf, 2003). Pero también despertó interés las transformaciones de los barrios obreros, derivados del paradigma imperante (Rodríguez et al., 2008; Cerda, 2016).

Esta macro-política de industrialización por sustitución de importaciones llegó a su fin con el golpe de estado el 11 de septiembre de 1973. Lo que vino a continuación y hasta el retorno a la democracia en 1990 fue una radical transformación de la sociedad y del espacio urbano en específico. Las siete modernizaciones del Estado llevadas a cabo por la dictadura militar desembocaron en una total desregulación del territorio y una apertura al mercado libre, abriendo paso a la globalización y a la inserción del país en la economía mundial. Todo esto a través de la especialización en exportaciones de ciertos productos como el cobre, la industria salmonera, la celulosa y el sector agroalimentario; sepultando la gran diversidad de industrias existentes a esa fecha, las cuales, incapaces de competir en una economía globalizada, prácticamente desaparecieron de la escena productiva urbana.

En la actualidad, la transformación política-económica que inauguró la dictadura, y que orbitó en los sucesivos gobiernos democráticos, convive con una nueva macro-política proyectada desde el Estado a inicios del siglo XXI, pero siempre con la anuencia de los círculos cercanos al poder empresarial y político.

La estrategia para la innovación comienza tempranamente con la implementación de una iniciativa alojada en el Ministerio de Economía, la Iniciativa Científica Milenio, que permitió inyectar recursos en diversas áreas orientadas a fomentar la perseguida innovación. En 2001 se implementó el Programa de Desarrollo e Innovación Tecnológica. La CORFO creó, por su parte, en 2004, el Fondo Innova. En 2005 se creó el Consejo Nacional de Innovación para la Competitividad por decreto supremo del presidente Ricardo Lagos, cuyo propósito fue elaborar una estrategia nacional de innovación. A pesar de ser una estrategia del Ministerio de Economía, rápidamente involucró al Ministerio de Educación y la CONICYT (Comisión Nacional de Ciencia y Tecnología) para solventar la inversión en recursos y la formación de capital humano avanzado.

Esta estrategia, junto con el fortalecimiento de la innovación empresarial a través de varios instrumentos financieros, tuvo como propósito la elaboración de clusters con un fuerte componente territorial. Esto último, dado que el debate teórico reitera la necesidad de incorporar al territorio regional en la innovación (Méndez, 2002). El espacio, entonces, juega un rol concluyente en la innovación y, para este efecto, la ciudad es un agente activo en dicho proceso, así lo plantea Paula Vera:

En este sentido planteamos, a modo de hipótesis, que el imaginario urbano-tecnológico en torno a la innovación influye material y simbólicamente en las transformaciones urbanas contemporáneas, delineando estrategias de marketing urbano, espacializando prácticas sociales, promoviendo estilos de vida, alentando ciertas subjetividades y construyendo determinados fragmentos espaciales dentro de la ciudad. (2017: 210) 
Dentro de la dimensión espacial de la innovación se ha construido una serie de supuestos donde cobra vital importancia el tránsito hacia ciudades creativas; con espacios de calidad, asumiendo que, presumiblemente, lo anterior incidirá en producir un ambiente laboral más proclive para el surgimiento de nuevas ideas. En palabras de Tan Yigitcanlar y Melih Bulu:

Se afirma que la calidad de vida y el lugar es un atractivo clave para los trabajadores del conocimiento que buscan diversidad de oportunidades, ideas y comodidades, tal como el acceso a espacios verdes, cultura y arte, y fuentes de creatividad e innovación, que les brindan un alto nivel de bienestar (Yigitcanlar \& Bulu, 2016: 6)

Es por esta razón por la que los conceptos asociados a la innovación y la creatividad han sido una preocupación desarrollada principalmente en la geografía urbana, pero últimamente también lo ha sido dentro de la geografía económica. En el primer caso, la geografía urbana ha abordado estos conceptos relacionándolos con la clase creativa (Peck, 2005) creatividad, innovación y ciudad (Lee \& Rodríguez-Pose, 2014); y espacialidad y desarrollo de la industria creativa (Hutton, 2006), entre otros. Sin embargo, ha sido la geografía económica, fundamentalmente, la que ha vinculado la innovación, la creatividad y el conocimiento a la dimensión territorial. Los estudios que se han llevado a cabo relacionan temáticas tales como la clase creativa y el crecimiento regional (Boschma \& Fritsch, 2009); la movilidad de esta clase creativa (Hansen \& Niedomysl, 2009); el conocimiento, talento y contexto en la utilidad de la clase creativa (Asheim \& Hansen, 2009); industrias creativas y sus efectos en el crecimiento urbano (Stam , De Jong , \& Marlet, 2008); clusters creativos, empresa y calidad del lugar (Heebels \& van Aalst, 2010).

Por otro lado, hay estudios que revelan la importancia para las regiones de generar elementos atractivos para estas profesiones creativas, ya que su competitividad aumentaría (Hansen \& Niedomysl, 2009); otros parten del análisis geográfico de la clase creativa y sus impactos en el desarrollo regional y cuestionan la aplicación de las ideas de Florida ${ }^{5}$ al contexto urbano (Asheim \& Hansen, 2009). Las opiniones divergentes ya habían surgido hacia 2005 , con un estudio de Jamie Peck (2005) donde señala que, en el actual contexto neoliberal, los términos de clase creativa o ciudades creativas en su despliegue performativo de innovación cultural liberal, no quiebran la hegemonía y orientación de las políticas urbanas existentes. Estas están basadas en la competencia inter-local; el mercado de lugares, el desarrollo impulsado por la propiedad y el mercado, la gentrificación y la desigualdad socio-espacial normalizada.

En resumen, los temas de la innovación, ciudades y territorios creativos hacen referencia, en primer lugar, a los clusters que se generan en la actualidad en donde las industrias del conocimiento juegan un rol preponderante. En segundo término, se enmarcan en las ciudades creativas y las comunidades que se forman en ellas, pero, sobre todo, en los efectos que estas nuevas ideas tienen en el espacio: la gentrificación y las políticas urbanas (Matus, 2017). 


\section{Valdivia y el caso de los Barrios Bajos}

Valdivia se sumó tempranamente al proceso de industrialización, el cual fue de alguna manera inducido por una ola de migrantes provenientes de Alemania, quienes rápidamente desarrollaron industrias de diverso tipo; así lo destacan diversos autores (Almonacid, 2013; Rodríguez et al., 2008; Guarda, 2001). A lo que Patricio Bernedo Pinto (1999) destaca que, más que una industrialización masiva, lo ocurrido en Valdivia fue un periodo manufacturero alcista. Es cierto que al comenzar el siglo XX, la ciudad era un pujante centro industrial, ubicado en la rivera de los ríos Calle-Calle y Valdivia, beneficiándose del sistema fluvial para el traslado de los insumos y productos finales de la industria (Saelzer Canouet \& Urbina, 2015). Una de las industrias relevantes en los Barrios Bajos era la curtiembre y fábrica de calzados Weiss, junto con diversos astilleros, y un grupo de pequeñas industrias, las cuales edificaron al menos un par de conjuntos de viviendas para dar respuesta al problema habitacional de la época (Rodríguez et al., en prensa).

Este barrio se dividió rápidamente en dos áreas específicas: la primera corrió tangente al río, la calle General Lagos, donde se ubicaron las viviendas de los industriales inmigrantes alemanes con viviendas de mayor tamaño. El segundo, fue al interior de la calle General Lagos, denominado los Barrios Bajos, donde se alojaron las viviendas de los trabajadores de las industrias que habían empezado a llegar a la ciudad (Araya Riquelme \& Saelzer Canouet, 2017), junto con establecimientos educacionales (ver Figura $\mathrm{N}^{\circ} 1$ ).

Figura $\mathrm{N}^{\circ} 1$.

Industrias y barrios obreros de los Barrios Bajos

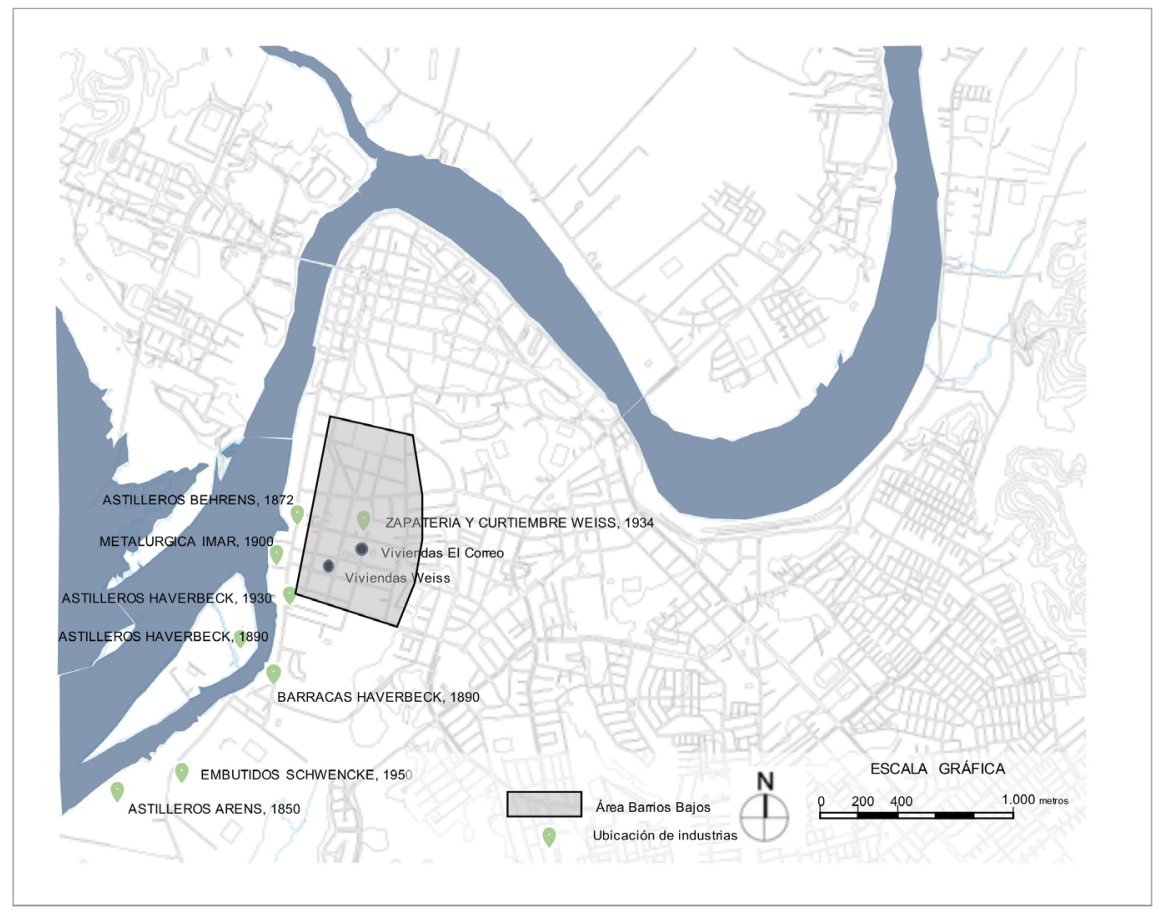

Fuente: elaboración propia 
Hacia 1956 la ciudad entendería la necesidad de crear su propia universidad, fundándose la Universidad Austral de Chile, que, en conjunto con una sede de la Universidad Técnica del Estado, formarían a los estudiantes de la educación superior de la ciudad (Almonacid Zapata, 2003). La enseñanza, derivada de las necesidades de la industria, fue un equipamiento de alta relevancia para esta época.

La vivienda fue otro aspecto relevante en este periodo dadas las necesidades habitacionales, que fueron de extrema urgencia para las crecientes poblaciones migrantes desde el campo a la ciudad. Por entonces los barrios obreros albergaban solo equipamientos básicos, ya que dependían de gran medida del paternalismo industrial para solventar sus necesidades, es decir no contaban con adecuada accesibilidad (calles de tierra o callejones), sin alcantarillado, ni electrificación, tal como da cuenta un diario local ${ }^{6}$.

A causa de las múltiples inundaciones del barrio, este siempre tuvo graves problemas de calles anegadas, situación que se vio agravada por la ausencia de veredas. Adicionalmente a la menoscabada vida urbana de los obreros y sus viviendas, tampoco fueron los espacios públicos una posibilidad de mejora en su calidad de vida; las plazas y parques no se constituyeron en una preocupación para la autoridad de ese período, de ello da cuenta la prensa de la época ${ }^{7}$. De hecho, los humedales de antaño se convertirian, a raíz de tener la peor calidad de suelo en la ciudad, en emplazamientos destinados a la vivienda social (Hidalgo et al., 2018); situación que, de acuerdo con los autores, se revertirá notablemente en las primeras décadas del siglo XXI, cuando la cercanía con la naturaleza -específicamente la relación con los humedales- abrirá un nicho de renta. Con esto la plusvalía de los sectores cercanos a estos espacios naturales subirá el interés de los sectores inmobiliarios. Lo anterior está directamente relacionado con la macro-política de innovación (ver más adelante) y que directamente se traduce en una visión diametralmente distinta respecto de los valores económicos que la naturaleza puede tener para el sector inmobiliario (Hidalgo et al., 2018).

Con el crecimiento expansivo de la ciudad a consecuencia de las políticas implementadas por la dictadura, aumentó el suelo urbano disponible. Los Barrios Bajos fueron quedando en el peri-centro de la ciudad y sufrieron una lenta, pero progresiva transformación. La presión sobre el precio del suelo para edificar departamentos y cabañas durante la década de los años noventa, permitió responder a la demanda para alojamiento de los estudiantes, todo ello fue señalando las orientaciones que seguiría el barrio. Un arreglo sostenido del problema del alcantarillado, el cual fue abordado por los gobiernos desde 2000 hasta 2012 (Lepe Núñez, 2014) y la pavimentación de calles y veredas condujeron a subir los precios del suelo progresivamente. Tal como puede ser visto en Rodríguez, Barría y Silva, (en prensa) donde la plusvalía de un conjunto de viviendas obreras en el centro de los Barrios bajos aumentó exponencialmente de precio. De acuerdo con los datos obtenidos del Conservador de Bienes Raíces de Valdivia, en la calle Phillippi \# 1433, propiedad inserta en el conjunto habitacional del Diario El Correo, se aprecia el incremento en la plusvalía de la propiedad (ver cuadro $\mathrm{N}^{\circ} 1$ ).

Diario El Correo de Valdivia, 28-12-1938, p. 9

Diario El Correo de Valdivia, 23-4-1948, p. 8. 13-10-1954, p.8. 16-10-1954, p.7 
Cuadro $N^{\circ} 1$.

Precio de propiedad en calle Phillippi \# 1433

\begin{tabular}{|l|l|}
\hline \multicolumn{1}{|c|}{ Año } & \multicolumn{1}{c|}{ Precio de venta } \\
\hline 17 de abril de 1979 & $\$ 160.000$ \\
\hline 6 de julio de 1979 & $\$ 180.000$ \\
\hline 1 de diciembre de 1982 & $\$ 381.000$ \\
\hline 31 de enero de 1990 & $\$ 3.200 .000$ \\
\hline 2 de enero de 2006 & $\$ 17.915 .090$ \\
\hline
\end{tabular}

Fuente: RODRIGUEZ, L. BARRIA, T. SILVA, R. Conjunto habitacional del Diario El Correo de Valdivia: Un breve relato urbano. Revista AUS, 2021. En prensa.

En la actualidad esta propiedad, a pesar de no estar en venta, su precio de mercado bordea los 80 millones de pesos. Lo anterior da una idea aproximada de la rentabilidad del sector, aun cuando es bastante heterogéneo en su edificación.

Con el advenimiento de la macro-política de innovación, que se sustenta en una serie de premisas estipuladas previamente, comenzó un proceso sostenido de transformación de las viviendas. Las otrora casas de industriales pasaron a ser viviendas patrimoniales que por su tamaño fueron convirtiéndose, algunas de ellas, en equipamiento vinculado a la educación superior. Otras casas patrimoniales e incluso instalaciones industriales de los Barrios Bajos pasaron a formar parte de la oferta hotelera. Tal es el caso de la Casa Pausenberger (ver figura $N^{\circ} 2$ ). Lo propio ha ocurrido con algunos conjuntos de viviendas obreras, lo cual amplía la visión de patrimonio, abarcando no solo lo monumental, sino también las peculiaridades del habitar y la memoria (Brito et al., 2018).

Figura $\mathrm{N}^{\circ} 2$

Hotel boutique en la antigua Casa Pausenberger

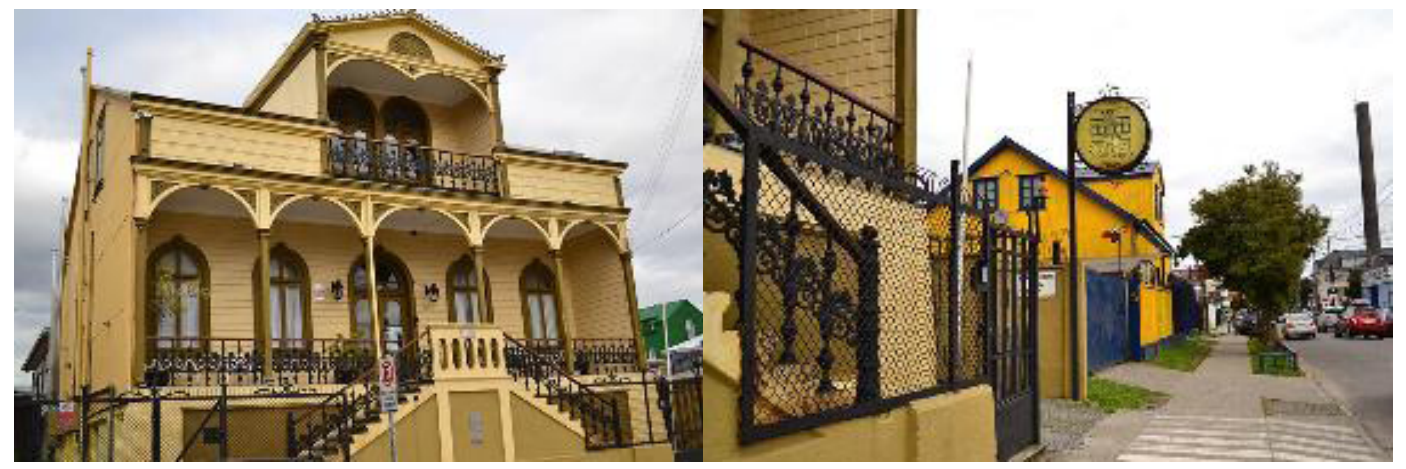

Fuente: elaboración propia, archivo de autora.

El espacio urbano fue así adaptándose a los nuevos tiempos, fenómeno que también puede ser apreciado en otras latitudes, tal como Lígia Isabel Paz Mendes Oliveira observa en el caso de Palo Alto en Barcelona (2015). La autora destaca que, el proceso de reconversión industrial se encaminó hacia un centro creativo e incorporó la iniciativa empresarial, la producción cultural, la valorización del patrimonio y la creación de nuevos espacios públicos. Lo anterior se menciona ya que el origen industrial del sector en Barcelona ha dado paso a un barrio enfocado a las industrias 
creativas, lo cual refrenda la idea de una relación entre el pasado histórico industrial y los nuevos desenvolvimientos en los Barrios Bajos.

En los Barrios Bajos, el financiamiento que vino desde el Estado, a través de sus recursos humanos, financieros, y técnicos, permitió la instalación de galerías de arte, oficinas de co-working, arquitectura y diseño, locales gastronómicos y servicios en general asociados a la industria cultural; todos ellos pasaron a formar parte del paisaje urbano del sector conviviendo con las otrora viviendas obreras (ver figura $\mathrm{N}^{\circ} 3$ ).

Figura $\mathrm{N}^{\circ} 3$

Comercio gastronómico en los Barrios Bajos

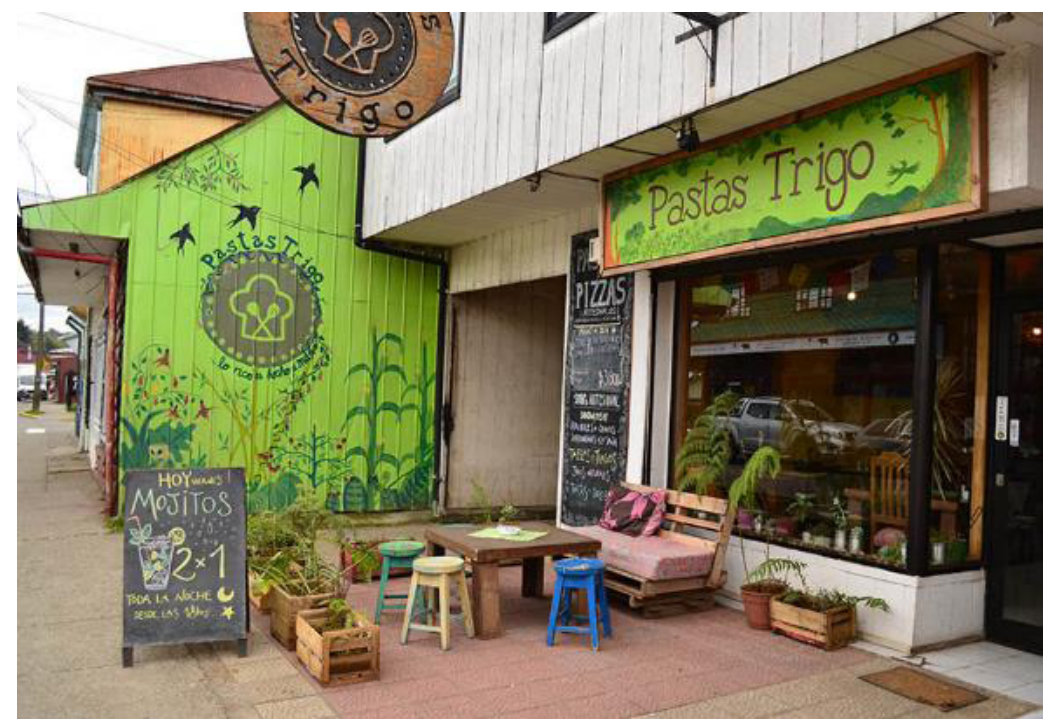

Fuente: elaboración propia, archivo de autora.

En virtud de aquello, iniciativas de diversa envergadura comenzaron a surgir y transformaron la atmosfera de lo que antes fue exclusivamente un barrio obrero-industrial, para convertirse progresivamente en un barrio en proceso de adaptación al marco ideológico en desarrollo, al convivir con algunos elementos conspicuos de la cultura urbana contemporánea chilena. La estética vintage del comercio comenzó a desplegar una aspiración nostálgica a partir de la utilización de objetos antiguos en la decoración. Con esto se recrea un ambiente amistoso y familiar con los nuevos y antiguos vecinos del lugar, sobre todo en algunas arterias, percibidas como poco seguras durante algunas horas de la noche. Para entender el surgimiento de esta estética del comercio, es necesario atender lo argumentado por Jordi Nofre, quien encuentra en Lisboa que la estética vintage higieniza la atmosfera del lugar.

El consumo de esta vida nocturna tradicional promovida recientemente como una nueva forma de distinción social también puede verse como la estrategia de (re) producción de una vida nocturna social y moralmente saneada que margina a todos los que se consideran inapropiados, o en otras palabras, socialmente peligrosos para la marca de la ciudad de Lisboa. (2013: 118). 
Los Barrios Bajos se ha transformado progresivamente, como se da cuenta en el texto, $y$ las demandas de los nuevos vecinos -dentro de estos un grupo de gente llamada clase creativa- incorporan también nuevos modos de desplazamiento. Para este efecto se construyen dos ciclo-vías, una terminada a inicios de 2018 y otra en 2019 (Ver figura №4).

Dentro de los nuevos vecinos que han llegado al sector se encuentran Elisa Figueroa y Gabriela Urrutia, ambas son las propietarias de la Galería Barrios Bajos y el café La Contienda de Rosales, ubicados en la calle Pérez Rosales n. ${ }^{\circ}$ 986. En 2016 se les adjudicó un proyecto CORFO ${ }^{8}$. Desde entonces han estado trabajando incesantemente en la galería y el café que funcionan en el mismo recinto, pero separadamente. El lugar queda a unas cuadras del centro de la ciudad, en una arteria muy concurrida de los Barrios Bajos, donde se aprecia en la vereda opuesta al recinto un grupo de departamentos construidos hacia los años noventa. Estos poseen un sistema estructural prefabricado en acero con un reticulado diagonal, lo que hizo que la gente los asociara con edificios rusos.

La galería de arte tiene una fachada metálica desplegable que esconde una antigua vivienda, a la cual se accede a través de dos entradas; una da acceso a la galería y la otra al café. Como la vereda en esa cuadra posee una platabanda -entre la vereda y la calle- de tamaño considerable, comparado con otras del vecindario bastantes reducidas, en ese lugar se localiza una pequeña terraza, construida por las dueñas del emprendimiento.

Hace dos años que tienen la galería funcionando y un año que viven en las casas pareadas que edificaron en el fondo del sitio. Para ellas la galería es su "proyecto de vida", tal como lo expresan en reiteradas ocasiones durante la entrevista. En función de lo anterior, ellas han hecho múltiples esfuerzos por vincularse con los vecinos; han organizado actividades en el club de box del barrio, las juntas de vecinos, así como también han realizado encuestas con el propósito de conocer el grado de conocimiento de los vecinos respecto de la galería. De acuerdo con lo que ellas mismas dicen, la mayoría de los vecinos encuestados sabía de la presencia de la galería, sin embargo, nunca habían entrado, aun cuando consideran importante poder tener contacto con la cultura. Las galeristas piensan que el café contribuirá a romper esa distancia que las separa de los vecinos y que el café ha servido un poco para este efecto. Hoy viene gente a almorzar al café y de paso se introduce en la galería para ver las exposiciones.

La relación de la galería con el vecindario ha sido lenta. Pero esto también ha servido para cuestionarnos la relevancia del arte. Por ejemplo, a veces invitamos a adultos mayores del barrio para conversar acerca de la memoria, esas son acciones más direccionadas (entrevista a Gabriela Urrutia).

También comentan que la instalación de la Facultad de Arquitectura y Artes, UACH, a pocas cuadras del emprendimiento cultural, ha permitido que los profesores de las distintas especialidades artísticas se acerquen a participar de las iniciativas llevadas a cabo en este lugar. Al indagar acerca de la razón por la que eligieron vivir y trabajar en Barrios bajos Elisa Figueroa dice:

Proyecto FRAGUA (CORFO): Programa de encuentro y desarrollo para el emprendimiento y la innovación de la industria creativa de las artes visuales. 
Barrios Bajos nos acomoda como mujeres, como vecinas y esto al final es un rubro donde tu pones todo el cuerpo. Al final, como dice la Gaby, [el barrio] está súper precarizado; tienes que estar ahí con todo, entonces también el que pudiéramos vivir y trabajar en el mismo lugar tenía mucho sentido. A nosotras nos hace mucho sentido vivir acá porque es un barrio que nos interesa. Como mamá, en el caso de la Gaby, la plaza está acá [plaza Pastene]. El trabajo que desarrollamos con la galería significa también poder encontrarse de forma súper directa con las personas [con las] que uno trabaja (Entrevista a Elisa Figueroa $\mathrm{C} / \mathrm{P})$.

Ya estando aquí, instaladas en este lugar, se ganaron el proyecto CORFO, y este incluyó la realización de charlas, talleres y algunas exposiciones. De acuerdo con las entrevistadas, un gran logro del proyecto fue la visibilidad que alcanzaron, que les permitió hacer redes y, por esto mismo, las han contactado otros artistas para exponer en la galería. En el recuento de por qué ellas creen que ganaron el proyecto, son categóricas al afirmar que la razón que las llevó a que se lo adjudicasen, fue estar alineadas con la estrategia regional de promoción de las industrias creativas. A partir de eso, y una vez concluido el proyecto, todo el tiempo transcurrido desde entonces ha sido autogestionado, evidenciándose un cierto desgaste producido por la necesidad de estar permanentemente en la consecución de recursos.

A pesar de los financiamientos invertidos por el Estado para desarrollar la innovación, esta aún se muestra vulnerable a los vaivenes propios de la labor creativa. El esfuerzo desplegado por los beneficiarios, como las galeristas entre muchos otros, se introduce en una lógica de autogestión en un sector de pocos recursos dentro de la economía.

Todo el mundo habla de las industrias creativas, pero la mayor parte de las veces, esta sigue generando empleos informales, precarios. Entonces el desarrollo de la industria creativa no es tal como es el caso de la industria minera, por ejemplo. Entonces todos hablan de la industria del cine, pero la verdad es que también la industria del cine es sumamente precaria y lo va a seguir siendo seguramente. (Entrevista a Gabriela Urrutia C/P).

Las entrevistadas creen que, aun cuando ellas estaban alineadas con la política regional de industrias creativas, en realidad aún nadie sabía mucho a que se referían con el desarrollo de las industrias creativas, ni mucho menos cómo estas se iban a gestionar. En opinión de las entrevistadas, este sector al seguir generando empleos precarios y estar basado fuertemente en la autogestión, se traduce en un gran desgaste para quienes están en la innovación y la creatividad en los emprendimientos.

\section{Los impactos de corto y largo plazo de las macro-políticas}

Desde todos los puntos de vista, la versatilidad de la vivienda en los Barrios Bajos ha permitido nuevos usos. Lo anterior permite ir visualizando el surgimiento de pequeños cluster relativos a la industria cultural (ver figura $\mathrm{N}^{\circ} 4$ ). También se observa que, si bien los proyectos que han recibido financiamiento del Estado no son muchos, existe una diferenciación entre ellos, los que recibieron financiamiento Corfo se localizan en edificaciones mayores, tal como las casas de los industriales y los proyectos Sercotec y Sercotec Semilla, más bien al interior de los barrios Bajos. Aun así, se observa una interrelación entre las instituciones que han recibido financiamiento y otro tipo de equipamientos acordes al modelo. 
Figura $\mathrm{N}^{\circ} 4$.

Mapa de localización de las instalaciones e infraestructura asociada a la innovación

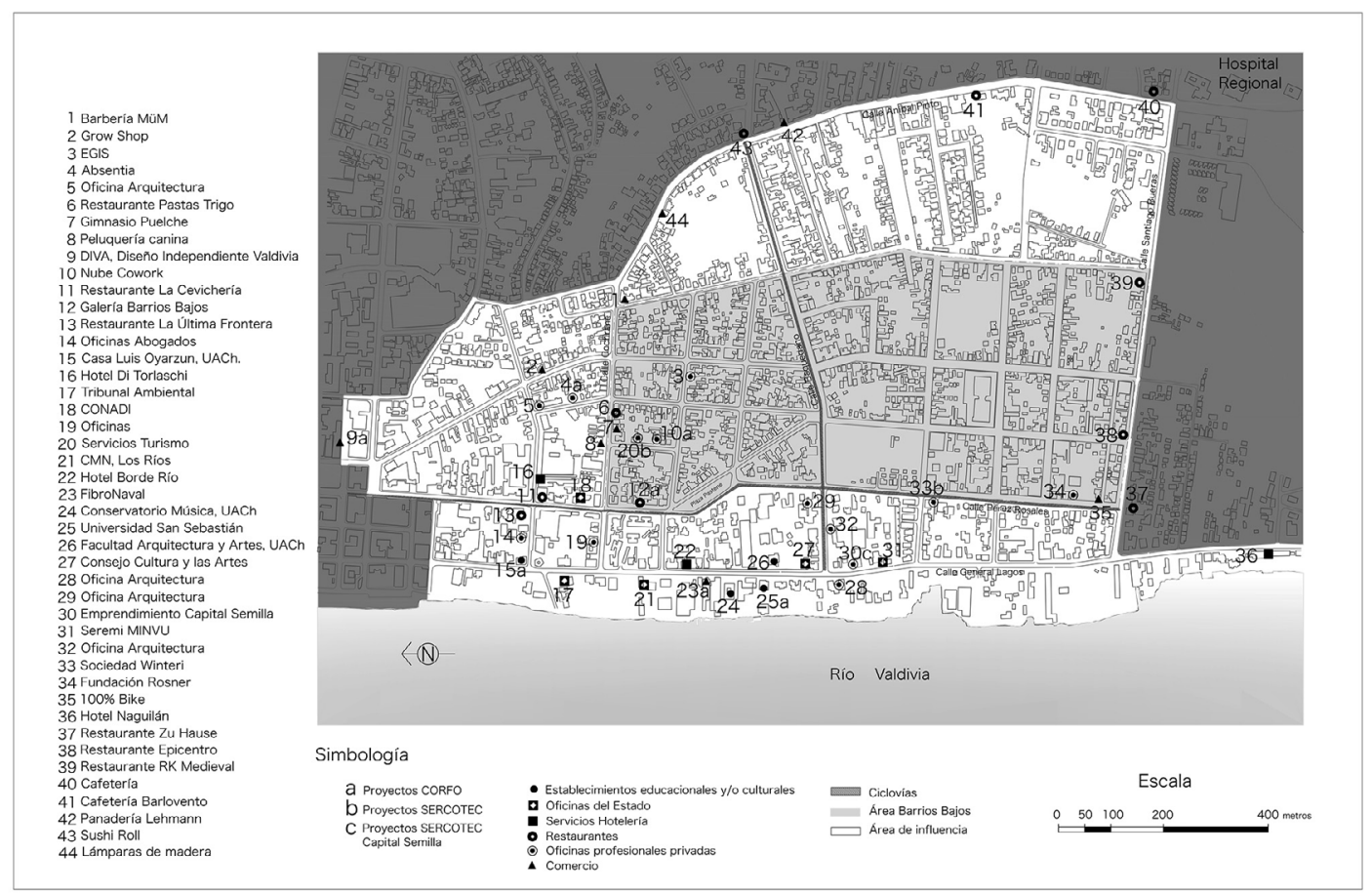

Fuente: elaboración propia.

El sistema de transporte, otrora favorecedor exclusivo del vehículo motorizado, ahora acoge también, mediante las ciclo-vías, a los nuevos usuarios del barrio. Sin duda los sistemas de saneamiento han mejorado la calidad de vida del barrio en su conjunto y han permitido un mejor desplazamiento peatonal por el barrio.

En una primera lectura las casas patrimoniales de la calle General Lagos; algunas convertidas en equipamiento de educación superior, oficinas gubernamentales (especialmente en el caso de la Universidad Austral de Chile y el Estado) y equipamiento vinculado a las artes, la cultura y los servicios asociados (ver figura $\mathrm{N}^{\circ} 5$ ).

La educación se ha especializado y ha dado lugar a nuevas áreas de innovación, pero también de creación. Nuevas formas de realizar la función laboral, en casos de oficinas de trabajo compartidas (coworking) y colaborativo se han ubicado en los interiores del barrio. El comercio y los servicios vinculados a este nuevo paradigma se han ubicado en ejes más transitados y donde también se aprecia la adaptación de antiguas viviendas, tal como el uso de antiguas industrias para satisfacer una demanda más exigente de servicios. Los vecinos ahora pueden hacer uso del espacio público, el cual se ha vuelto relevante considerando el rol progresivamente importante que han adquirido los espacios verdes y la naturaleza urbana (ver figura $N^{\circ} 6$ ). A continuación, se observa que la transformación del barrio se produce en un proceso de adaptación, donde conviven los antiguos usuarios y sus edificaciones, junto con los nuevos, quienes adaptan las propiedades en función de sus requerimientos. 
Figura $\mathrm{N}^{\circ} 5$.

Instalaciones mayores en calle General Lagos

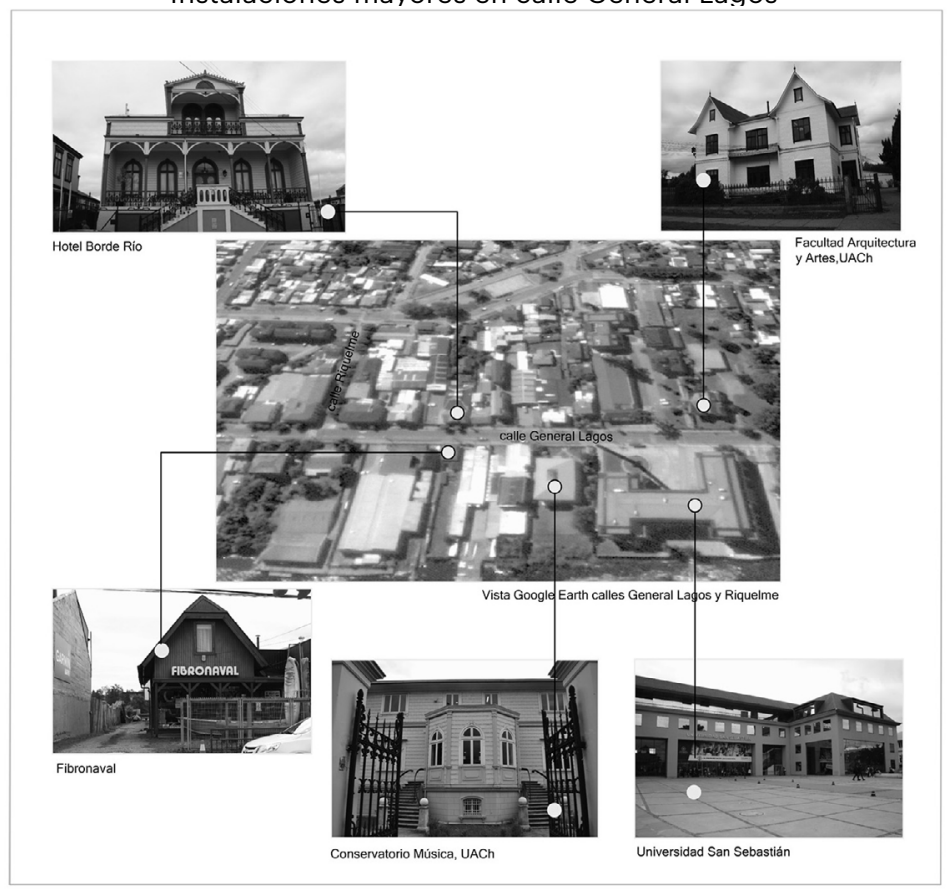

Fuente: elaboración propia en base a una imagen central de Google Earth.

Figura $\mathrm{N}^{\circ} 6$.

Comercio asociado a la macro-política de Innovación

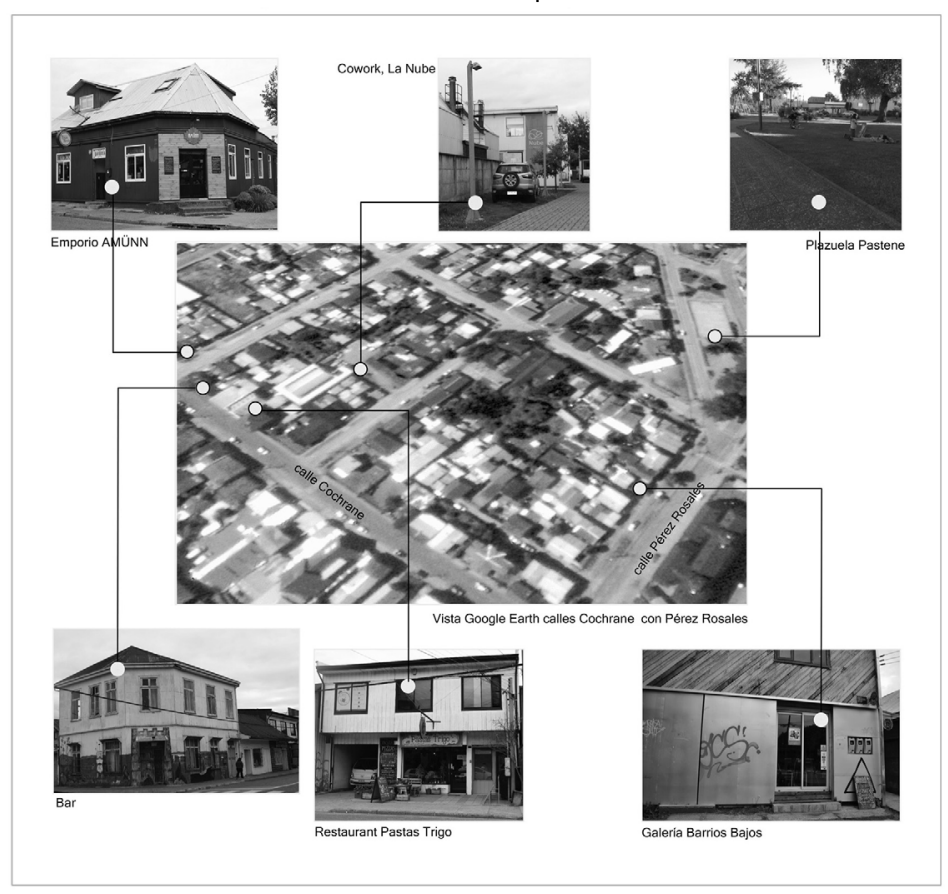

Fuente: elaboración propia en base a una imagen central de Google Earth. 
Por último, el cuadro n. ${ }^{\circ} 2$ compara diversos elementos del espacio urbano que diferencian las macro-políticas de industrialización por sustitución de importaciones y la de innovación; a partir de esto se reseñan algunas reflexiones.

Cuadro $\mathrm{N}^{\circ} 2$.

Comparación espacial de macro-políticas de industrialización por sustitución

de importaciones e innovación

\begin{tabular}{|c|c|c|c|c|}
\hline $\begin{array}{l}\text { industrialización } \\
\text { por sustitución }\end{array}$ & $\begin{array}{c}\text { Programa de edifica- } \\
\text { ciones }\end{array}$ & Infraestructura & $\begin{array}{c}\text { Desplazamien- } \\
\text { to }\end{array}$ & equipamiento \\
\hline $\begin{array}{l}\text { de importacio- } \\
\text { nes } \\
\text { Comienzo del } \\
\text { siglo XX-década } \\
1970\end{array}$ & $\begin{array}{l}\text { Provisión de vivienda } \\
\text { obrera } \\
\text { Vivienda de industriales } \\
\text { Industrias }\end{array}$ & $\begin{array}{l}\text { Infraestruc- } \\
\text { tura escolar y } \\
\text { de enseñanza } \\
\text { superior }\end{array}$ & $\begin{array}{l}\text { Apertura y pa- } \\
\text { vimentación de } \\
\text { calles (adoqui- } \\
\text { nado) }\end{array}$ & $\begin{array}{l}\text { Electrificación } \\
\text { y alcantarillado. }\end{array}$ \\
\hline $\begin{array}{l}\text { Innovación } \\
\text { Siglo XXI en } \\
\text { adelante }\end{array}$ & $\begin{array}{l}\text { Espacio laboral colabo- } \\
\text { rativo } \\
\text { Espacio publico } \\
\text { Servicios adyacentes }\end{array}$ & $\begin{array}{l}\text { Espacio inves- } \\
\text { tigación-desa- } \\
\text { rrollo e innova- } \\
\text { ción. }\end{array}$ & $\begin{array}{l}\text { Construcción } \\
\text { de ciclo-vías }\end{array}$ & $\begin{array}{l}\text { Mejoramiento } \\
\text { del sistema de } \\
\text { aguas lluvias }\end{array}$ \\
\hline
\end{tabular}

Fuente: elaboración propia.

Tal como se muestra en el cuadro, las macro-políticas producen una transformación en términos de elementos urbanos, lo cual orienta el desarrollo de los Barrios Bajos hacia una dinámica distinta de la inicial. De acuerdo con el tiempo que ha pasado entre cada una de estas macro-políticas, incorporando la dictadura, aun cuando esta no ha sido objeto de análisis en esta investigación directamente, podemos decir que el lapso es de alrededor de tres décadas para que entren en vigencia.

\section{Conclusiones}

El efecto que tienen las macro-políticas en el caso de Los Barrios Bajos se observan tanto en el sistema de la vivienda, los equipamientos urbanos, los servicios, el espacio público, como en la movilidad. Si bien es cierto que comienzan tímidamente a localizarse nuevos usuarios, nuevos tipos de instalaciones, con el tiempo este proceso de adaptación se vuelve observable en el lugar. Por ello es por lo que la política pública reviste un especial interés a partir de la forma en la cual esta va organizando el espacio para adecuarse a sus requerimientos. Sin embargo, es prudente decir que las manifestaciones urbanas producidas por anteriores macro-políticas continúan conviviendo con las nuevas instalaciones de la política actual. En términos generales podemos decir que se produce una adaptación política-espacial convivencial.

El Estado, por su parte, proporciona ciertos recursos y los inyecta en el espacio por la vía de diversos instrumentos que fomentan la dinámica económica del lugar (Alvarado Peterson, 2019). Junto con lo anterior, el sector privado se beneficia mediante el acoplamiento espacial al proceso, modificando lenta, pero progresivamente, la estructura urbana del sector e incorpora nuevos vecinos y usuarios de los servicios y equipamientos. 
La adaptación producida para satisfacer los requerimientos de cada nueva macro-política va utilizando -en algunos casos- la infraestructura existente, la cual se presume entra en una categoría de nueva funcionalidad, a veces diametralmente distinta respecto de aquella para la que fue originalmente edificada. Lo anterior hace que no cualquier barrio pueda entrar en dialogo con las nuevas políticas, ya que se presume unas condiciones preexistentes apropiadas para la adaptación al marco ideológico imperante.

La educación sigue teniendo una presencia significativa dentro del barrio, aun cuando en el tránsito histórico esta se hace más compleja al incorporar entidades de educación superior, beneficiarias de las edificaciones existentes y que proceden de la época industrial (viviendas e instalaciones). En este mismo sentido, la otrora vivienda de clase trabajadora comienza lentamente un proceso de mejoramiento al transformar las estructuras familiares del barrio.

Los servicios e instalaciones de orden más público, que satisfacen los requerimientos de las transformaciones del barrio, se ubican en las arterias más transitadas, las cuales bordean el límite imaginario de los Barrios Bajos. Esto último deja entrever que deben subsistir satisfaciendo los requerimientos tanto de los nuevos usuarios, como de los antiguos.

La innovación se posiciona adecuadamente en este barrio debido a que este es un barrio histórico de antigua vocación industrial de Valdivia, que actualmente cuenta con edificaciones que fueron construidas largo tiempo atrás y que son versátiles en sus usos. Lo anterior hace que sea menos dificultoso la instalación de la nueva macro-política de innovación. Tal como fue en el caso de la macro-política de industrialización por sustitución de importaciones, el origen del barrio habría estado asociado a la cercanía al centro de la ciudad y del aparato productivo, llámese industrias, beneficiarias por su cercanía al río, en la actualidad se debe a la presencia de patrimonio residencial e industrial (entendiendo el patrimonio en un amplio sentido y no en el convencional vinculado al edificio monumental). Por último, la misma cercanía al centro de la ciudad, especialmente para quienes optan por otros medios de movilidad, atrae a nuevos usuarios, quienes valoran los atributos de este barrio.

\section{Referencias}

ALISTE ALMUNA, E.; CONTRERAS ALONSO, M. \& SANDOVAL MANRÍQUEZ, V. Industrialización, desarrollo y ciudad: transformaciones socio-demográficas y espaciales en la geografía social del gran Concepción (1950-2010). Revista INVI, 2012, Vol. 27, Nº75, p. 21-71. http://revistainvi.uchile.cl/ index.php/INVI/article/view/635

ALMONACID ZAPATA, F. La industria valdiviana en su apogeo (1870-1914), Valdivia: Ediciones Universidad Austral de Chile, 2013.

ALMONACID ZAPATA. F. Historia de la Universidad Austral de Chile (1954-2003), Valdivia: Ediciones Universidad Austral de Chile, 2003.

ALMONACID ZAPATA, F. Ideas y proyectos en torno a la vivienda obrera en la Ciudad de Valdivia, 1900-1941. Revista Austral de Ciencias Sociales, 2000, №4, p. 81-114. http://doi:10.4206/rev.austral.cienc.soc.2000.n4-05 
ALVARADO PETERSON, V. El bienestar en el Estado neoliberal: escenarios de la propiedad en el Gran Santiago. Cultura.-hombre-sociedad, 2019, Vol.29, N², p. 13-35. http://dx.doi.org/10.7770/07192789.2019.cuhso.04.a02.

ASHEIM, B. \& HANSEN, HK. Knowledge Bases, Talents, and Contexts: On the Usefulness of the Creative Class Approach in Sweden. Economic Geography, 2009, Vol. 85, N4, p. 425-442. https:// doi.org/10.1111/j.1944-8287.2009.01051.x

ARAYA RIQUELME, P. \& SAELZER CANOUET, G. Restauración e imaginario urbano: algunas observaciones antropológico-arquitectónicas en torno a la idea de patrimonio. Revista Arquitecturas del Sur, 2017, Vol. 35, N51, p. 20-31. http://revistas.ubiobio.cl/index.php/AS/article/view/2624

BERNEDO PINTO, G. Los industriales alemanes de Valdivia, 1850-1914. Revista de Historia, 1999, №32, p. 5-42. http://www.memoriachilena.gob.cl/602/w3-article-98537.html

BORSDORF, A. Cómo modelar el desarrollo y la dinámica de la ciudad latinoamericana. EURE, 2003, Vol. 29, № 86, p. 37-49. http://www.eure.cl/index.php/eure/article/view/1265

BOSCHMA, RA.; FRITSCH, M. Creative Class and Regional Growth: Empirical Evidence from Seven European Countries. Economic Geography, 2009, Vol. 85, N4, p. 391-423. https://doi.org/10.1111/ j.1944-8287.2009.01048.x

BRITO, A. CERDA, G. FUENTES, P. \& PEREZ, L. Industria y habitar colectivo, conjuntos habitacionales en el sur de Chile. Santiago: STOQ editorial, 2018.

CARIOLA SUTTER, C. \& SUNKEL, O. Un siglo de historia económica de Chile: 1830-1930. Madrid: Ediciones Cultura Hispánica del Instituto de cooperación Iberoamericana, 1982.

CASANOVA BRITO, M. La centro-izquierda, el corporativismo empresarial y las contradicciones internas del Estado desarrollista en Chile, 1932-1954. Izquierdas, 2019, №48, p.190-210. http:// dx.doi.org/10.4067/S0718-50492019000400190.

CERDA, G. Población obrera Isla Teja de Valdivia, 1939. la acción modernizadora de la caja del seguro obrero obligatorio. Revista AUS, 2016, ํ20, p 42-47. https://doi.org/10.4206/aus.2016. n20-07

CORFO. Geografía económica de Chile Tomo III. Santiago de Chile: DIBAM, Centro de Investigaciones Diego Barros Arana, 2013 (ed. original 1963).

CRUZ, A. La ruta de la innovación en Chile. Journal of Technology Management \& Innovation, 2008, Vol. 3, N¹, p. 1-9. https://www.jotmi.org/index.php/GT/article/view/curr4/433

GUARDA, G. Nueva historia de Valdivia. Santiago: Ediciones Universidad Católica de Chile, 2001.

HANSEN, HK, NIEDOMYSL, T. Migration of the creative class: evidence from Sweden. Journal of Economic Geography, 2009, Vol. 9, N², p. 191-206. https://doi.org/10.1093/jeg/lbnO46 
HEEBELS, B. \& VAN AALST, I. Creative clusters in Berlin: Entrepreneurship and the quality of place in Prenzlauer Berg and Kreuzberg. Geografiska Annaler Series B-Human Geography, 2010, Vol. 92B, N4, p. 347-363. https://doi.org/10.1111/j.1468-0467.2010.00357.x

HIDALGO, R.; RODRÍGUEZ, L. \& ALVARADO, V. Arriba del cerro o sobre el humedal: producción de naturaleza y expansión inmobiliaria en ciudades marinas y fluviales. el caso de Valparaíso y Valdivia, chile. Diálogo andino, 2018, N56, p. 87-100. https://dx.doi.org/10.4067/S071926812018000200087

HIDALGO DATTWYLER, R.; SANTANA, D. \& ALVARADO PERTESON, V. Mitos, ideologías y utopías neoliberales de la producción del espacio: hacia una agenda de investigación alternativa. En: En las costas del neoliberalismo. Serie geolibros $\mathrm{N}^{\circ} 23$, Santiago: Ediciones Pontificia Universidad Católica, 2016, p. 24-66.

HIDALGO DATTWYLER, R. \& JANOSCHKA, M. La ciudad Neo liberal. Gentrificación y exclusión en Santiago de Chile, Buenos Aires, Ciudad de México y Madrid. Santiago de Chile: Serie Geolibros No 19, Instituto de Geografía, Pontificia Universidad Católica de Chile / Departamento de Ciencia Política y Relaciones Internacionales, Universidad Autónoma de Madrid, 2014.

HURTADO RUIZ-TAGLE, C. Concentración de población y desarrollo económico. El caso chileno, Santiago: Universidad de Chile, 1966.

HUTTON, T. Spatiality, built form, and creative industry development in the inner city Environment and Planning A, 2006, Vol. 38, Nº10, p. 1819-1841. https://doi.org/10.1068/a37285

ITURRA MUÑOZ L. El uso de la fotografía para problematizar lo urbano. Transferencias metodológicas, etnografía visual en la enseñanza de arquitectura. Revista de Arquitectura, 2015, Vol. 19, No28, p. 22-30. http://doi:10.5354/0719-5427.2015.37080

LEE, N. \& RODRIGUEZ-POSE, A. Creativity, cities, and innovation. Environment and Planning A, 2014, Vol. 46, N5, p. 1139-1159. https://doi.org/10.1068/a46202

LEPE NÚÑEZ, R. Memorias de los inundados del Centro de Valdivia, tesis para optar al título de Pedagogía en Historia y Ciencias Sociales. Universidad Austral de Chile, 2014. http://cybertesis. uach.cl/tesis/uach/2014/ffl594m/doc/ffl594m.pdf

MATTOS, C.A. de. Santiago de Chile, globalización y expansión metropolitana: lo que existía sigue existiendo. Eure. Revista de Estudios Urbano Regionales, 1999, Vol. 25, N76, p. 29-56. https://scielo.conicyt.cl/scielo.php?script=sci_arttext\&pid=S0250-71611999007600002

MATUS, C. Estilos de vida e imaginarios urbanos en nuevos residentes de Lastarria y Bellas Artes: el barrio patrimonial como escenario de diversidad, distinción y movilidad. Eure. Revista de Estudios Urbano Regionales, 2017, Vol. 43, №129, p. 165-186. http://dx.doi.org/10.4067/S025071612017000200008 
MÉNDEZ, R. Innovación y desarrollo territorial: Algunos debates teóricos recientes. Eure. Revista de Estudios Urbano Regionales. 2002, Vol. 28, N84, p. 63-83. http://dx.doi.org/10.4067/S025071612002008400004.

MOYA MUÑOZ, P. y MOLINA JARA, F. Innovación y emprendimiento en el discurso político chileno. Journal of Technology Management \& Innovation, 2017, Vol. 12, №1, p. 93-99. http://dx.doi. org/10.4067/S0718-27242017000100010

NOFRE, J. "Vintage Nightlife”: Gentrifying Lisbon downtown. Fennia - International Journal of Geography, 2013, Vol. 191, №2, p. 106-121. https://fennia.journal.fi/article/view/8231

OLIVEIRA, L.I.P.M. Culture as an engine in Palo Alto's urban regeneration process. On the W@ terfront, 2015, Vol. 37, $\mathrm{N}^{\circ} 0$, p. 7-45. https://revistes.ub.edu/index.php/waterfront/article/ view/18817/21480

ORTEGA MARTÍNEZ, L. La crisis de 1914-1924 y el sector fabril en chile. Revista Historia, 2012, Vol. 45, No2, p. 433-454. http://dx.doi.org/10.4067/S0717-71942012000200003

PECK J. Struggling with the creative class. International Journal of Urban and Regional Research, 2005, Vol. 29, N4, p. 740-770. https://doi.org/10.1111/j.1468-2427.2005.00620.x

PÉREZ, M. "A new poblador is being born": Housing struggles in a gentrified area of Santiago. Latin American Perspectives, 2017, Vol. 44, N³, p. 28-45. doi: https://doi.org/10.1177\%2F0094582X16668318

RODRIGUEZ, L. BARRIA, T. \& SILVA, R. Conjunto habitacional del Diario El Correo de Valdivia: Un breve relato urbano. Revista AUS, 2021. En prensa.

RODRÍGUEZ N., L.; CORDERO J., E.; RODRÍGUEZ J., G. Y GUTIÉRREZ, C. La desestructuración de un barrio industrial en la crisis de la modernidad valdiviana, Chile. Revista de Geografía Norte Grande, 2008, N40, p. 59-76. http://dx.doi.org/10.4067/S0718-34022008000200004

SAELZER CANOUET, G.E., URBINA, S.A. Urbanismo fluvial en el apogeo industrial de Valdivia: Desaparición y recuperación (ca. 1850-2012). Revista de Urbanismo, 2015, №33, p. 104-131. http:// doi10.5354/0717-5051.2015.34285

SANCHEZ, R. Las dinámicas urbanas. Revista Geografía. Norte Grande, 2013, N56, p.5-6. http:// dx.doi.org/10.4067/S0718-34022013000300001

SILVA, E. The Import-Substitution Model: Chile in Comparative Perspective. Latin American Perspectives, 2007, Vol. 34, N³, p. 67-90. www.jstor.org/stable/27648023

SILVA HIDALGO, R. Una forma de habitar en clave modernizadora. El barrio Arica de Valdivia, 1948-1960. Revista Sophia Austral, 2020, №25, p. 139-159. http://www.sophiaaustral.cl/index.php/ shopiaaustral/article/view/309 
STAM, E., DE JONG, JPJ. \& MARLET, G. Creative industries in the Netherlands: Structure, development, innovativeness and effects on urban growth. Geografiska Annaler Series B-Human Geography, 2008, Vol. 90B, N², p. 119-132. https://doi.org/10.1111/j.1468-0467.2008.00282.x

TAYLOR, M. From Pinochet to the 'third Way': Neoliberalism and Social Transformation in Chile. London: Pluto Press, 2006.

VERA, P. Procesos de recualificación urbana e imaginarios de la innovación. El caso de Rosario, Argentina. Eure. Revista de Estudios Urbano Regionales, 2017, Vol. 43, N¹29, p. 209-234. http:// mail.eure.cl/index.php/eure/article/view/1582/1010

VOS EYZAGUIRRE, B. de. El surgimiento del paradigma industrializador en Chile, (1875-1900) Santiago de Chile: DIBAM, Centro de Investigaciones Diego Barros Arana, 1999. http://www.memoriachilena.gob.cl/602/w3-article-8445.html

YIGITCANLAR, T. \& BULU, M. Urban Knowledge and Innovation Spaces. Journal of Urban Technology, 2016, Vol. 23, No1, p. 1-9. https://doi.org/10.1080/10630732.2016.1164443 
